\title{
Computational Investigation of the Electronic and Optical Properties of Planar Ga-Doped Graphene
}

\author{
Nicole Creange, ${ }^{1}$ Costel Constantin, ${ }^{1}$ Jian-Xin $\mathrm{Zhu},{ }^{2,3}$ \\ Alexander V. Balatsky, ${ }^{4,5}$ and Jason T. Haraldsen ${ }^{1}$ \\ ${ }^{1}$ Department of Physics and Astronomy, James Madison University, Harrisonburg, VA 22807, USA \\ ${ }^{2}$ Theoretical Division, Los Alamos National Laboratory, Los Alamos, NM 87545, USA \\ ${ }^{3}$ Center for Integrated Nanotechnologies, Los Alamos National Laboratory, Los Alamos, NM 87545, USA \\ ${ }^{4}$ Institute of Material Science, Los Alamos National Laboratory, Los Alamos, NM 87545, USA \\ ${ }^{5}$ Nordic Institute for Theoretical Physics, KTH Royal Institute of Technology and Stockholm University, Roslagstullsbacken 23, \\ 10691 Stockholm, Sweden
}

Correspondence should be addressed to Jason T. Haraldsen; j.t.haraldsen@unf.edu

Received 3 June 2015; Accepted 21 July 2015

Academic Editor: Sergei Sergeenkov

Copyright (C) 2015 Nicole Creange et al. This is an open access article distributed under the Creative Commons Attribution License, which permits unrestricted use, distribution, and reproduction in any medium, provided the original work is properly cited.

We simulate the optical and electrical responses in gallium-doped graphene. Using density functional theory with a local density approximation, we simulate the electronic band structure and show the effects of impurity doping (0-3.91\%) in graphene on the electron density, refractive index, optical conductivity, and extinction coefficient for each doping percentage. Here, gallium atoms are placed randomly (using a 5-point average) throughout a 128-atom sheet of graphene. These calculations demonstrate the effects of hole doping due to direct atomic substitution, where it is found that a disruption in the electronic structure and electron density for small doping levels is due to impurity scattering of the electrons. However, the system continues to produce metallic or semimetallic behavior with increasing doping levels. These calculations are compared to a purely theoretical $100 \%$ Ga sheet for comparison of conductivity. Furthermore, we examine the change in the electronic band structure, where the introduction of gallium electronic bands produces a shift in the electron bands and dissolves the characteristic Dirac cone within graphene, which leads to better electron mobility.

\section{Introduction}

Understanding the complex properties of materials is a fundamental goal for the advancement of technologies and other industries in the world today. Specifically, the examination and prediction of electronic properties and phase transitions in insulators and metals have gained much attention, typically for the investigations of exotic topological states and/or large symmetry-breaking regimes [1]. Topological materials in particular have been gaining a lot of attention due to their many surface properties [2]. Specifically, the study of the electronic properties of graphene and its interactions with substrates and other materials has been shown to exhibit many desired phenomena for technological devices $[3,4]$.

Graphene is a $\mathrm{sp}^{2}$-bonded 2D sheet of carbon atoms arranged in a honeycomb pattern (shown in Figure 1) [4-6].
The bonding and arrangement of atoms allow graphene to have a very large tensile strength [7] and interesting thermal properties $[8,9]$. When considering graphene's atomistic size and weight, it is stronger than steel [10]. Furthermore, the $\pi$-bonding throughout the lattice provides a high electron mobility [11, 12], where the high durability, strength, and conductivity make graphene a suitable choice for use in nanodevices and electronics. However, even though graphene has a high tensile strength, the ability for the material to be a great component for electronics is still unclear.

Currently, graphene is being investigated for use in electronics through surface deposition on metals and semiconductors, where the interactions of graphene and other atoms are hoped to produce enhancements to the overall electronic properties [13]. This enhancement is due to the presence of surface or topological states [1], which are critical to 

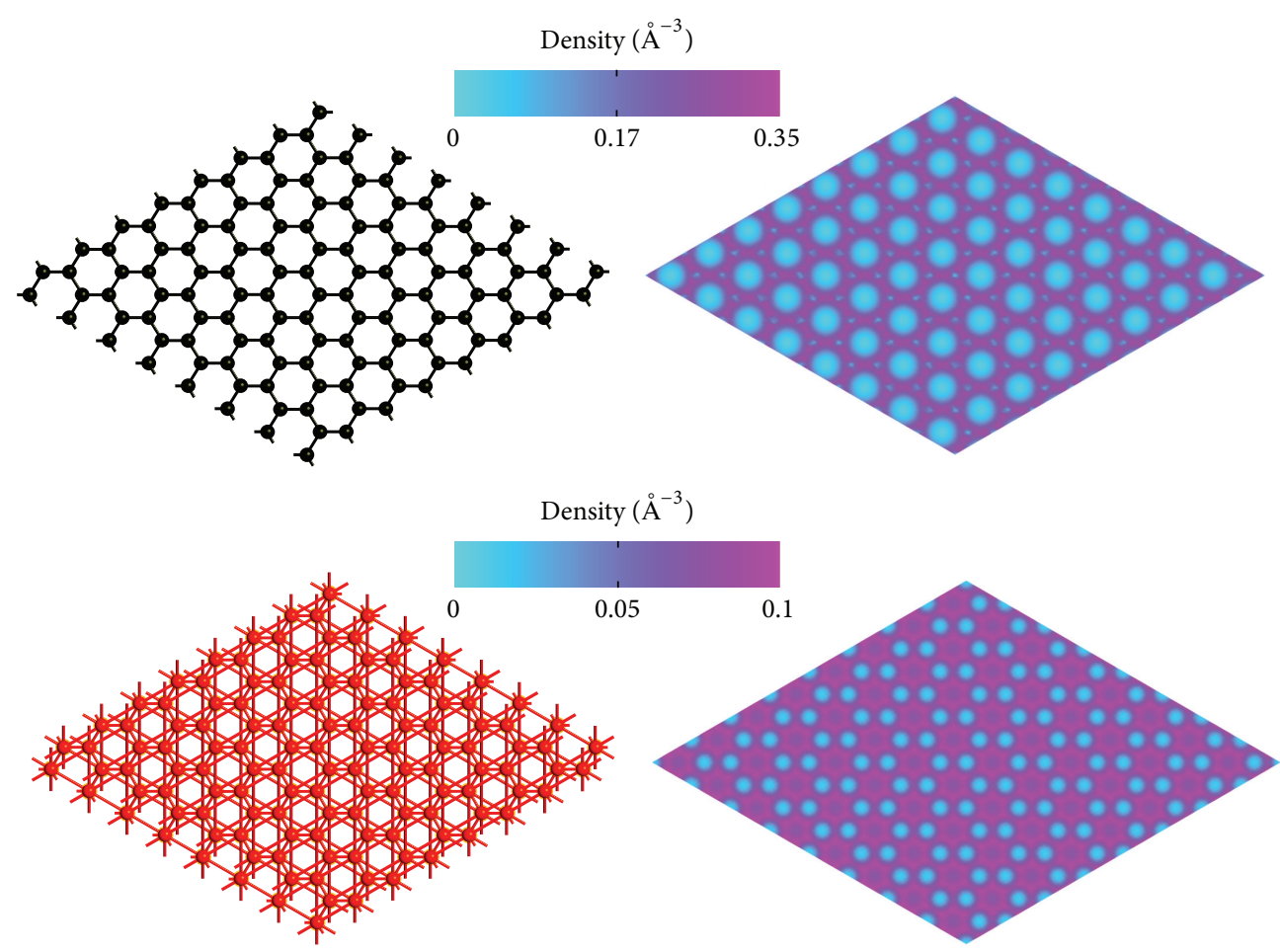

FIGURE 1: The structure (left) and calculated electron density (right) for a pure graphene sheet (top) and theoretical gallium sheet (bottom).

the understanding of the electron mobility in graphene, and has led to many researchers working to find ways for improving the electronic structure and overall electronic properties of graphene. While topological interactions with substrates and other materials play an important role in these advancements, the ability to change and control the electronic properties of graphene through direct substitution could provide a unique tunability to the mechanisms that drive technology further.

Recent experiments have demonstrated that it is possible to remove individual carbon atoms from the graphene lattice [14]. Furthermore, various experimental and theoretical papers have also shown or examined that carbon atoms can be replaced with other atoms (i.e., nitrogen, vanadium, chromium, manganese, cobalt, and iron) in specifically designed spots [15-20]. This opens a whole realm of possibilities for the tuning of the electronic properties of $2 \mathrm{D}$ materials through a manipulation of the electronic and topological states.

Topological states in graphene are produced through a unique conductivity point in its band structure known as the Dirac point, which typically allows electrons to easily move between the conduction and valence bands through a band structure Dirac cone at the Fermi level. The introduction of substituted or doped atoms will produce a breaking of the Dirac cone. However, the presence of these atoms will produce an impurity resonance at the Fermi level, which should provide an increase in the electron mobility and overall conductance. In many cases, the Dirac resonance has been examined using atoms that provide electron doping or magnetism [15-20]. Therefore, we propose the idea that impurity states may be hindered through hole doping or $\mathrm{p}$ type substitution in graphene using gallium atoms.

To examine the effects of topological states produced by p-substituted atoms, we examine the presence of directly substituted gallium atoms in graphene at low doping levels of $0-3.91 \%$. Using density functional theory in a local density approximation (LDA), we determine the electronic structure and optical properties for the various stages of $\mathrm{Ga}$ doping. Overall, we find that low doping of graphene with Ga will actually produce a decrease in the overall conductivity, which is a trend observed in the index of refraction and extinction coefficient as well. To reconcile these observations with known production of impurity resonances in Dirac materials, we examine the electron density and compare calculations to a theoretical 2D structure of pure Ga atoms. Here, we determine that the presence of a small amount of p-type dopant produces impurity scattering in the graphene sheet, which is the likely cause for the decrease in overall electron mobility.

\section{Computational Methodology}

We ran a series of density functional calculations using Atomistix Toolkit by Quantumwise [21-23]. In this study, we constructed a 128-atom supercell of graphene and subsequently replaced random carbon atoms with Ga using doping levels of $0,0.78,1.56,2.34,3.125,3.91$, and $100 \%$. This is illustrated in Figure 2. To simulate unbiased placement, $\mathrm{Ga}$ atom positions were determined by a random number generator, and, for systems with 2-5 impurity atoms, five different configurations were used to find the average quantities. 


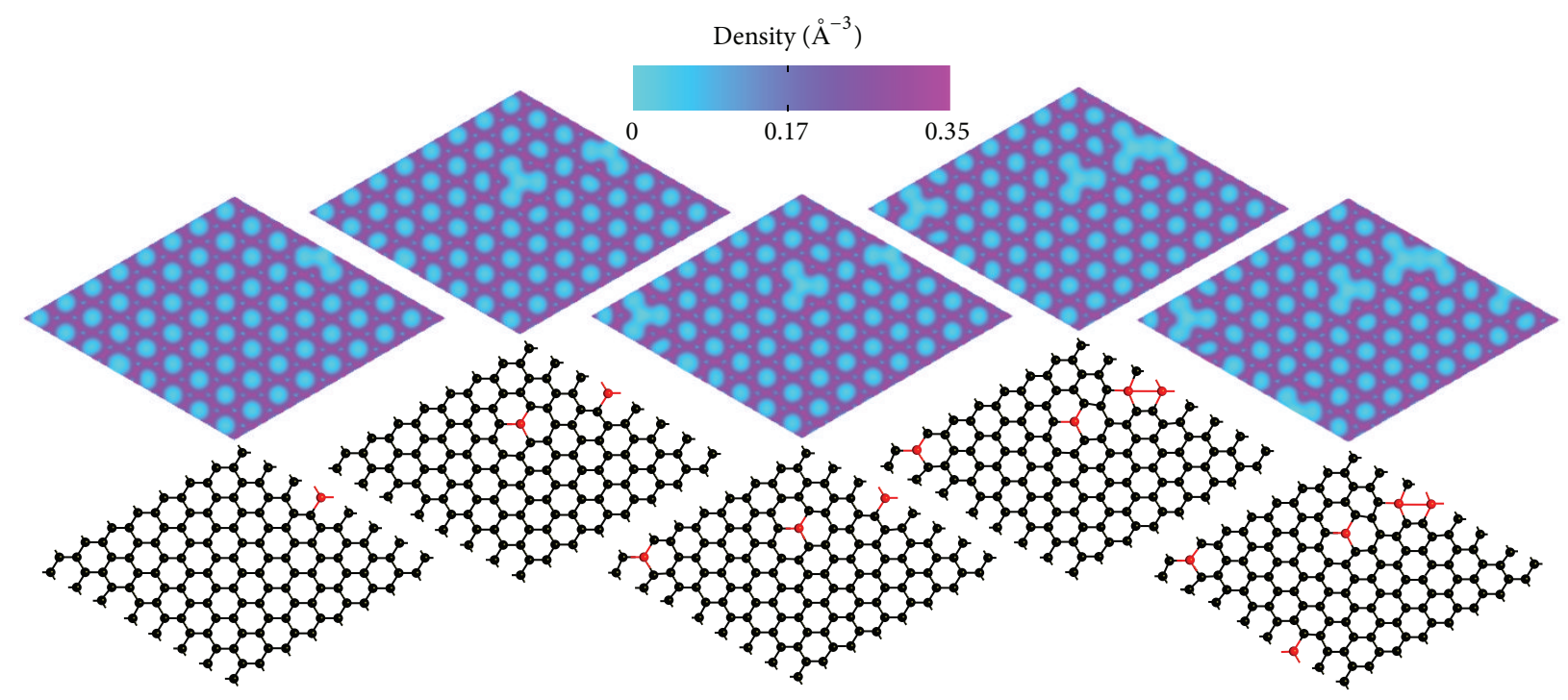

FIGURE 2: The calculated electron density as a function of spatial position in graphene with various concentrations of gallium (from left to right: $0.78,1.56,2.34,3.13$, and $3.90 \%$ Ga doping). The crystal structure is given directly below the density plots.

Using a local density approximation (LDA), a geometry optimization and band structure calculations were performed using a $k$-point sampling of $5 \times 5 \times 1$ with periodic boundary conditions for 23 different configurations. Furthermore, we determined the optical spectrum and electron density and calculated the conductivity, refractive index, and extinction coefficient. The doped graphene structure was kept in the planar configuration to allow us to examine only the electronic effects of substitution without energy parameters provided by lattice distortions. Further studies will analyze how the energy bands, conductivity, and optical properties are affected in the presence of a lattice distortion. However, the geometry optimization did produce a planar distortion due to the presence of the larger $\mathrm{Ga}$ atoms.

From the states in the electronic band structure and electron density [24], the Kubo-Greenwood formula used to calculate the susceptibility tensor from state $m$ to $n$ is

$$
\begin{aligned}
& \chi_{i j}(\omega)=-\frac{e^{2} \hbar^{4}}{m^{2} \epsilon_{0} V \omega^{2}} \\
& \cdot \sum_{n m} \frac{f\left(E_{m}\right)-f\left(E_{n}\right)}{E_{n}-E_{m}-\hbar(\omega-i \eta)}\left\langle n\left|j_{\alpha}\right| m\right\rangle\left\langle m\left|j_{\beta}\right| n\right\rangle,
\end{aligned}
$$

where $j_{\alpha}$ describes the momentum operator between matrix element between state $n$ and $m, V$ is the volume, $\eta$ is a finite broadening, and $f\left(E_{m}\right)=1 /\left(\exp \left(E / k_{B} T\right)+1\right)$ [25-27]. From the susceptibility tensor [26], we determine the real and imaginary parts of the dielectric tensor

$$
\epsilon_{r}(\omega)=(1+\chi(\omega))
$$

and the optical conductivity

$$
\sigma(\omega)=-i \omega \epsilon_{0} \chi(\omega) .
$$

From the dielectric tensor [27], the refractive index and extinction coefficient can be calculated as

$$
n=\sqrt{\frac{\sqrt{\epsilon_{1}^{2}+\epsilon_{2}^{2}}+\epsilon_{1}}{2}}
$$

and the extinction coefficient can be calculated as

$$
\kappa=\sqrt{\frac{\sqrt{\epsilon_{1}^{2}+\epsilon_{2}^{2}}-\epsilon_{1}}{2} .}
$$

Using these quantities, it is possible to evaluate the electron mobility of supercell.

\section{Results and Discussion}

Figure 3 shows the calculated electronic band structure for each configuration. This illustrates the dramatic change in electron mobility as $\mathrm{Ga}$ atoms are substituted into the graphene lattice. Once one $\mathrm{Ga}$ atom is substituted into graphene (Figure 3(b)), the characteristic Dirac cone shifts in energy and is broken and produces distinct bands at the Fermi level. As more atoms are substituted into the 2D lattice, the impurity bands form at zero energy which produces the observed impurity resonances.

From the calculated band structure, the dielectric tensor can be determined as a function of energy. Figure 4(a) shows the determined optical spectra for pure graphene and a theoretical analog of Ga atoms. This illustrates the expected increase in conductivity, which is characteristic of the simulated band structure.

Figure 5 shows the real and imaginary components of the calculated dielectric tensors for doping cases. The dielectric response shows a low energy shift as Ga is added to the system. From the calculated optical spectra, we can determine 


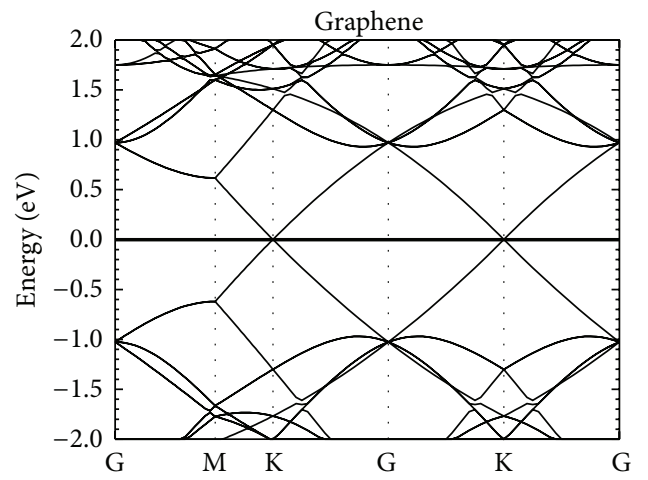

(a)

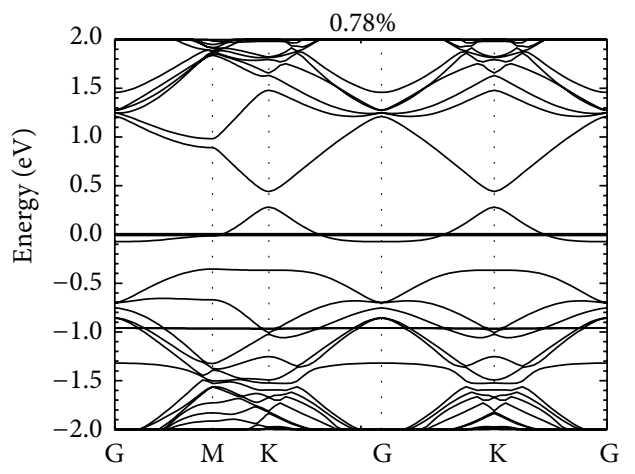

(b)

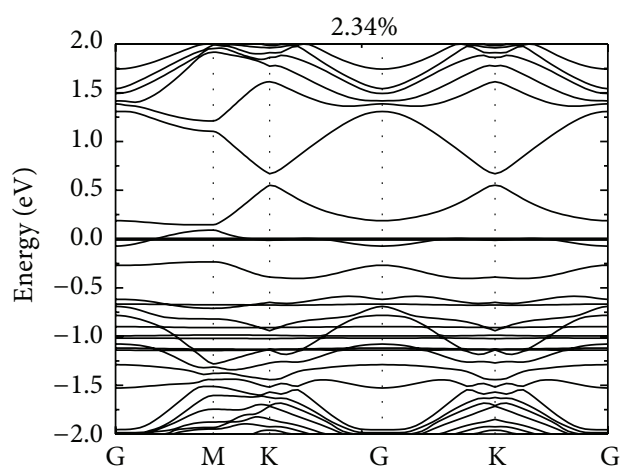

(d)

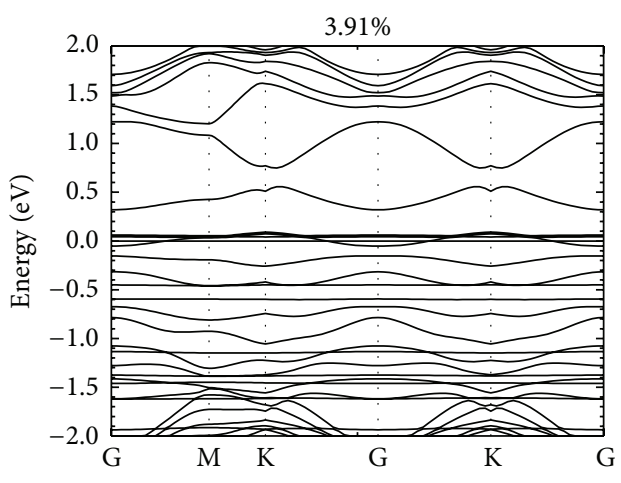

(f)

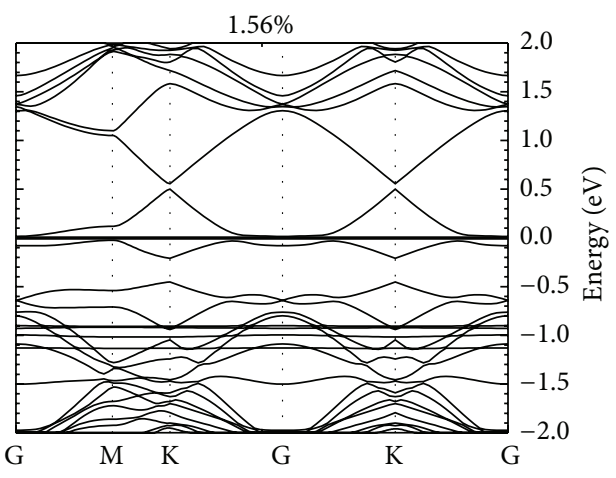

(c)

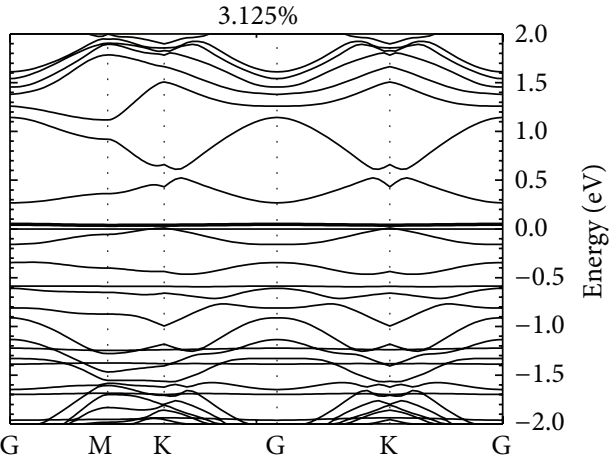

(e)

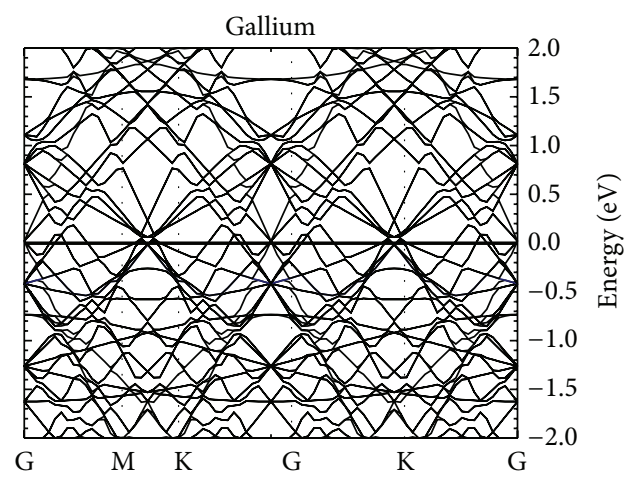

(g)

Figure 3: The simulated electronic band structure for graphene sheets with $0 \%$ (a), $0.78 \%$ (b), $1.56 \%$ (c), $2.34 \%$ (d), 3.13\% (e), 3.91\% (f), and $100 \%$ (g) substitution of gallium. 


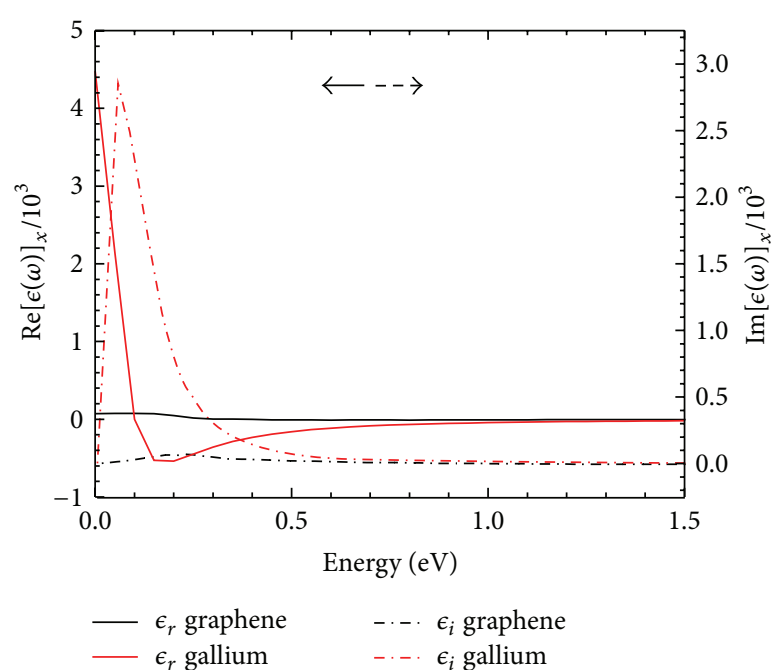

(a)

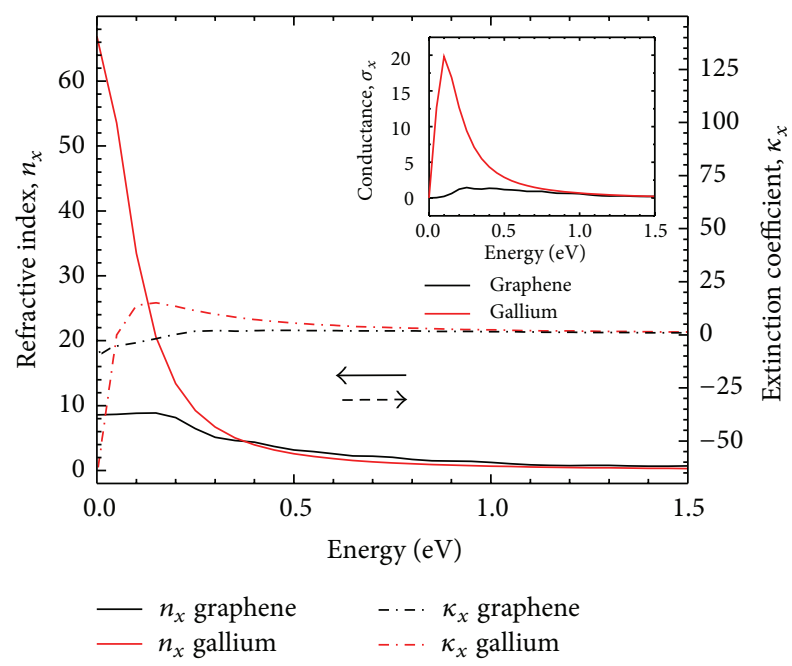

(b)

FIGURE 4: (a) Simulated dielectric response as a function of energy for a pure graphene sheet and a theoretical gallium sheet. (b) The calculated refractive index and extinction coefficient as functions of energy, where the conductance is placed in the inset.

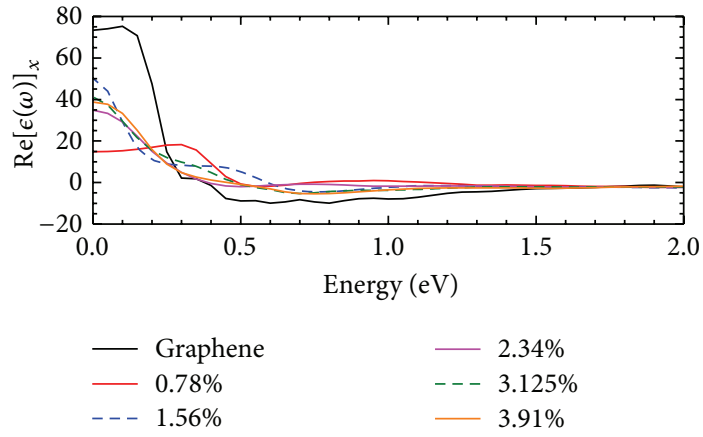

(a)

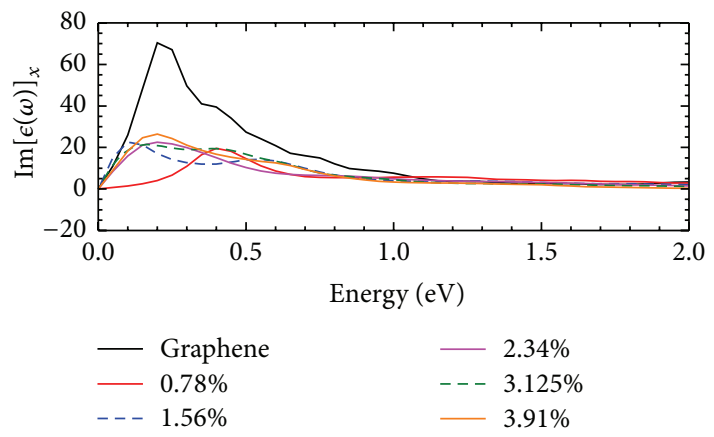

(b)

FIGURE 5: Simulated real (a) and imaginary (b) $x$-axis components of dielectric response for the various concentrations of gallium $(0,0.78$, $1.56,2.34,3.13$, and $3.91 \%)$. This is the average dielectric response consisting of a five-point configuration average. The average dielectric response along $y$-axis is similar.

the conductivity, refractive index, and extinction coefficient (shown in Figure 6).

From the comparison of the optical spectra properties for graphene and low doping cases of $\mathrm{Ga}$, there is a distinct loss of conductivity and electron mobility, which is in contradiction to the change in the optical properties from graphene to a pure Ga sheet. The optical properties also appear to be in slight contradiction to the electronic structure for the low doping Ga cases, where the impurity bands seem to provide crossover and conduction states at the Fermi level.

To understand these contradictions, we examined the electron density (shown in Figures 1 and 2) correlating to each configuration. From the electron density in the graphene sheet, it is shown that the electrons are distributed fairly evenly in between each carbon atom in the pure graphene sheet, where blue regions represent no or low electron density and red correlated to high electron density. However, when the first dopant is added, the electron density shifts away from this region, which is indicated by the large blue region.
This shift indicated the movement of the electrons from the gallium atoms. This trend continues through the low concentration Ga-doped sheets. However, in the theoretical pure gallium sheet, the electrons tend to cluster right on top of each gallium atom rather than in between the atoms, which produces the large increase in the electronic mobility.

As we have seen in both the optical spectrum and electron configurations, there is a general disturbance in the system causing a decrease in electron mobility. From the electron density, this decrease in conductivity seems to be due to impurity scattering at the low doping levels, which will restrict the ability to enhance the electronic properties through direct substitution. The formation of low electron density holes from the $\mathrm{Ga}$ atoms produces transmission pathway disruptions and as a gallium atom is added to the system, electrons encounter the holes in the graphene sheet and are scattered from them. This trend continues for all low doping percentages and the only change seems to occur at the pure gallium sheet. In this theoretical gallium sheet, 


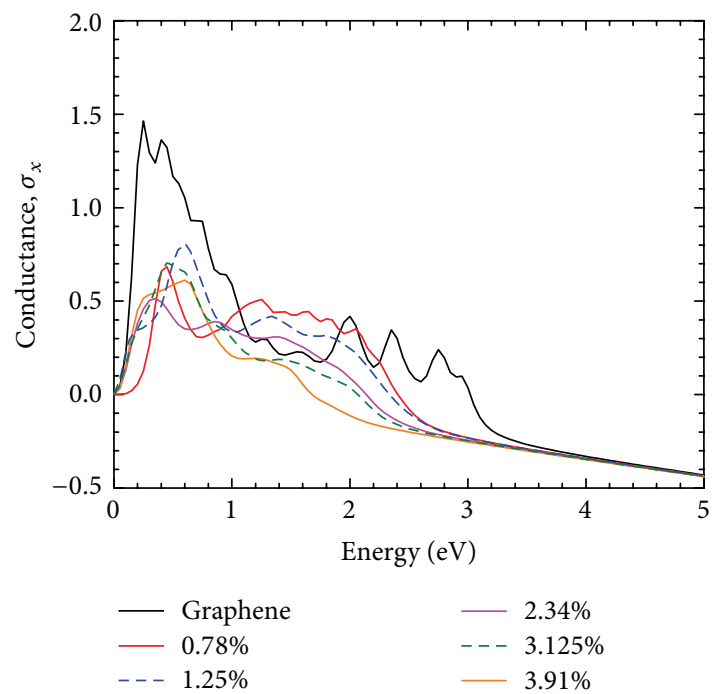

(a)

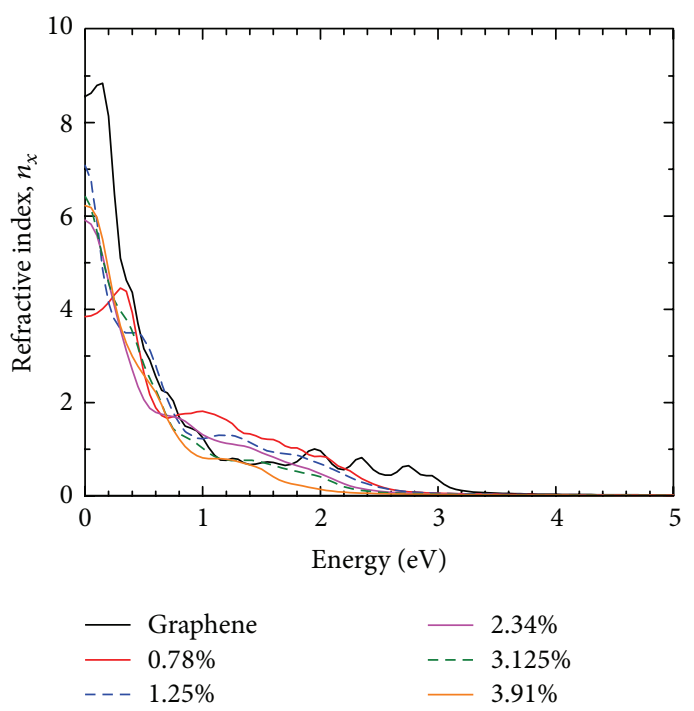

(b)

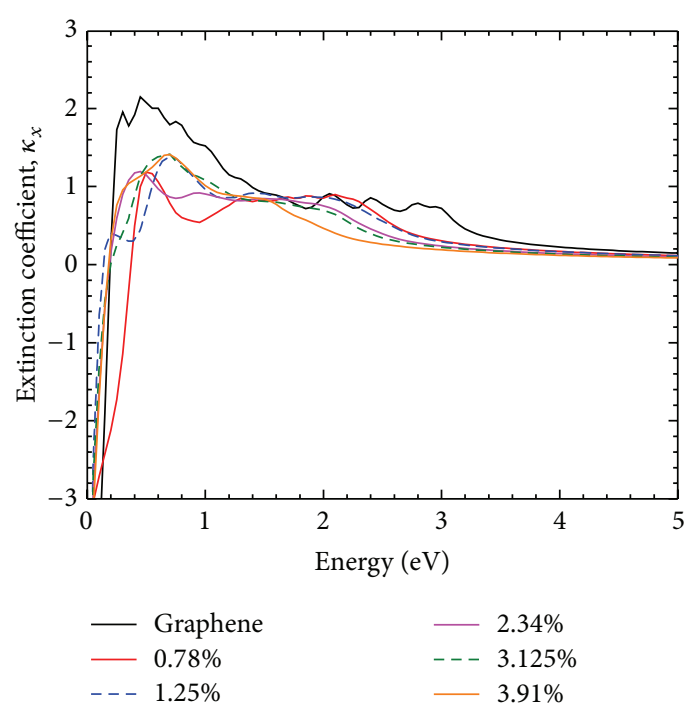

(c)

Figure 6: The calculated conductance (a), refractive index (b), and extinction coefficient (c) as function of energy for the various substitutions concentrations of gallium $(0,0.78,1.56,2.34,3.13$, and $3.91 \%)$.

the optical spectrum shows an extremely high conductance due to the disappearance of the impurity scattering from the uniformity of the sheet. The low to high conductivity from the doping cases to the theoretical Ga sheet indicates there must be a crossover point in the intermediate region of doping. However, when that region was calculated, the $2 \mathrm{D}$ sheet was found to be unstable in the geometry optimization. Therefore, it is only possible to examine the low doping regions to understand the possibility of enhanced electronic and optical properties.

\section{Conclusions}

In this study, we have investigated the effect of low percentage gallium doping on graphene in order to understand the possibility of electronic enhancement. Using density functional theory and a local density approximation, it was found that as Ga dopants are added to the system, there is a distinct shift in the optical spectrum that indicates a decrease in the conductance. Through an analysis of the electron for all configurations, it appears that this is due to impurity scattering at the $\mathrm{Ga}$ atomic site. This analysis was done in the confines of the planar geometry to minimize contributions produced by geometric distortions. Further work will incorporate $z$-axis graphene distortion to provide a better understanding of the system.

\section{Conflict of Interests}

The authors declare that there is no conflict of interests regarding the publication of this paper. 


\section{Acknowledgments}

Nicole Creange, Costel Constantin, and Jason T. Haraldsen acknowledge the support of the Institute for Materials Science at Los Alamos National Laboratory. The work at Los Alamos National Laboratory was carried out, in part, under the auspice of the U.S. DOE and NNSA under Contract no. DEAC5206NA25396 and supported by U.S. DOE Basic Energy Sciences Office (Alexander V. Balatsky). Work of Alexander V. Balatsky was also supported by European Research Council (ERC) DM 321031. This work was also, in part, supported by the Center for Integrated Nanotechnologies, a U.S. DOE Office of Basic Energy Sciences user facility (Jian-Xin Zhu).

\section{References}

[1] T. O. Wehling, A. M. Black-Schaffer, and A. V. Balatsky, "Dirac materials," Advances in Physics, vol. 63, no. 1, pp. 1-76, 2014.

[2] M. Klintenberg, J. T. Haraldsen, and A. V. Balatsky, "Computational search for strong topological insulators: an exercise in data mining and electronic structure," Applied Physics Research, vol. 6, no. 4, article 31, 2014.

[3] A. K. Geim and K. S. Novoselov, "The rise of graphene," Nature Materials, vol. 6, no. 3, pp. 183-191, 2007.

[4] A. H. C. Neto, F. Guinea, N. M. R. Peres, K. S. Novoselov, and A. K. Geim, "The electronic properties of graphene," Reviews of Modern Physics, vol. 81, no. 1, pp. 109-162, 2009.

[5] Y. Zhu, S. Murali, W. Cai et al., "Graphene and graphene oxide: synthesis, properties, and applications," Advanced Materials, vol. 22, no. 35, pp. 3906-3924, 2010.

[6] K. S. Novoselov, A. K. Geim, S. V. Morozov et al., "Two-dimensional gas of massless Dirac fermions in graphene," Nature, vol. 438, no. 7065, pp. 197-200, 2005.

[7] K. I. Bolotin, K. J. Sikes, Z. Jiang et al., "Ultrahigh electron mobility in suspended graphene," Solid State Communications, vol. 146, no. 9-10, pp. 351-355, 2008.

[8] E. Pop, V. Varshney, and A. K. Roy, "Thermal properties of graphene: fundamentals and applications," MRS Bulletin, vol. 37, no. 12, pp. 1273-1281, 2012.

[9] A. A. Balandin, S. Ghosh, W. Bao et al., "Superior thermal conductivity of single-layer graphene," Nano Letters, vol. 8, no. 3, pp. 902-907, 2008.

[10] N. Savage, "Materials science: super carbon," Nature, vol. 483, no. 7389, pp. S30-S31, 2012.

[11] C. Lee, X. Wei, J. W. Kysar, and J. Hone, "Measurement of the elastic properties and intrinsic strength of monolayer graphene," Science, vol. 321, no. 5887, pp. 385-388, 2008.

[12] S. V. Morozov, K. S. Novoselov, M. I. Katsnelson et al., "Giant intrinsic carrier mobilities in graphene and its bilayer," Physical Review Letters, vol. 100, no. 1, Article ID 016602, 2008.

[13] J.-H. Chen, C. Jang, S. Xiao, M. Ishigami, and M. S. Fuhrer, "Intrinsic and extrinsic performance limits of graphene devices on $\mathrm{SiO}_{2}$," Nature Nanotechnology, vol. 3, no. 4, pp. 206-209, 2008.

[14] K. K. Gomes, W. Mar, W. Ko, F. Guinea, and H. C. Manoharan, "Designer Dirac fermions and topological phases in molecular graphene," Nature, vol. 483, no. 7389, pp. 306-310, 2012.

[15] A. W. Robertson, B. Montanari, K. He et al., "Dynamics of single Fe atoms in graphene vacancies," Nano Letters, vol. 13, no. 4, Article ID 14681475, pp. 1468-1475, 2013.
[16] E. J. G. Santos, A. Ayuela, and D. Sánchez-Portal, "Firstprinciples study of substitutional metal impurities in graphene: structural, electronic and magnetic properties," New Journal of Physics, vol. 12, Article ID 053012, 2010.

[17] F. M. Hu, T. Ma, H.-Q. Lin, and J. E. Gubernatis, "Magnetic impurities in graphene," Physical Review B, vol. 84, Article ID 075414, 2011.

[18] H. Wang, Q. Wang, Y. Cheng et al., "Doping monolayer graphene with single atom substitutions," Nano Letters, vol. 12, no. 1, pp. 141-144, 2012.

[19] R. Lv, Q. Li, A. R. Botello-Méndez et al., "Nitrogen-doped graphene: beyond single substitution and enhanced molecular sensing," Scientific Reports, vol. 2, article 586, 2012.

[20] C. B. Crook, C. Constantin, T. Ahmed, J. Zhu, A. V. Balatsky, and J. T. Haraldsen, "Proximity-induced magnetism in transitionmetal substituted graphene," Scientific Reports, vol. 5, Article ID 12322, 2015.

[21] Atomistix ToolKit version 13.8, QuantumWise A/S, http://www .quantumwise.com/.

[22] M. Brandbyge, J.-L. Mozos, P. Ordejón, J. Taylor, and K. Stokbro, "Density-functional method for nonequilibrium electron transport," Physical Review B-Condensed Matter and Materials Physics, vol. 65, no. 16, Article ID 165401, 2002.

[23] J. M. Soler, E. Artacho, J. D. Gale et al., "The SIESTA method for ab initio order-N materials simulation," Journal of Physics Condensed Matter, vol. 14, no. 11, pp. 2745-2779, 2002.

[24] D. J. Griffiths, Introduction to Electrodynamics, Prentice Hall, 1999.

[25] P. B. Allen, Conceptual Foundations of Materials: A Standard Model for Ground- and Excited-State Properties, edited by: S. G. Louie, M. L. Cohen, chapter 6, Elsevier, Amsterdam, The Netherlands, 2006.

[26] R. M. Martin, Electronic Structure, Cambridge University Press, Cambridge, UK, 2004.

[27] W. A. Harrison, Solid State Theory, McGraw-Hill, 1970. 

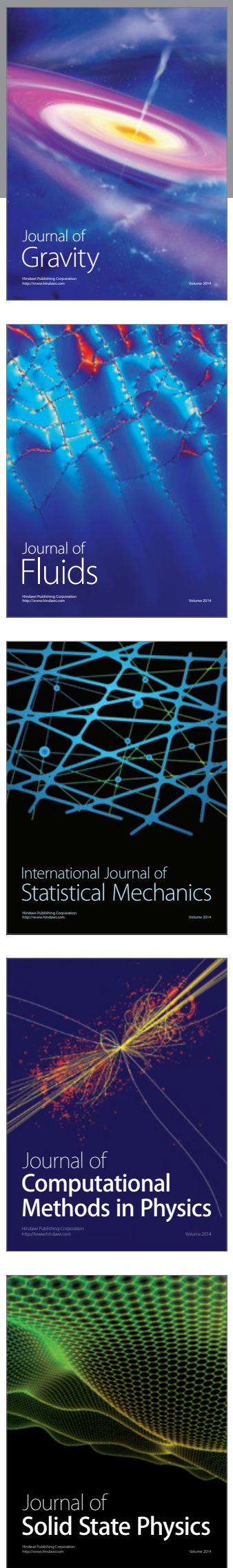

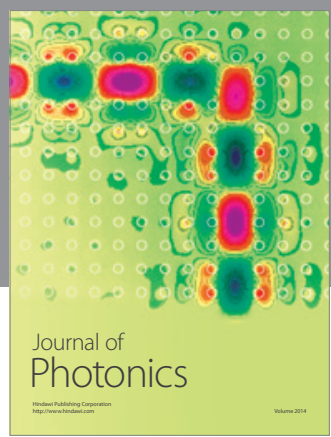

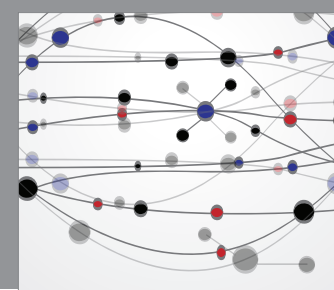

The Scientific World Journal

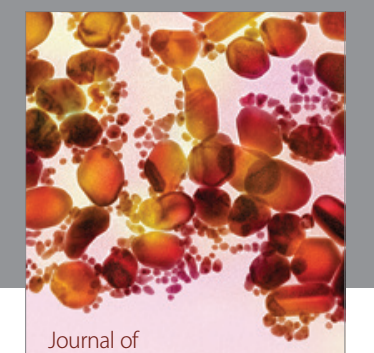

Soft Matter
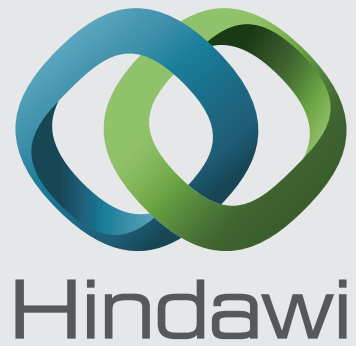

Submit your manuscripts at

http://www.hindawi.com
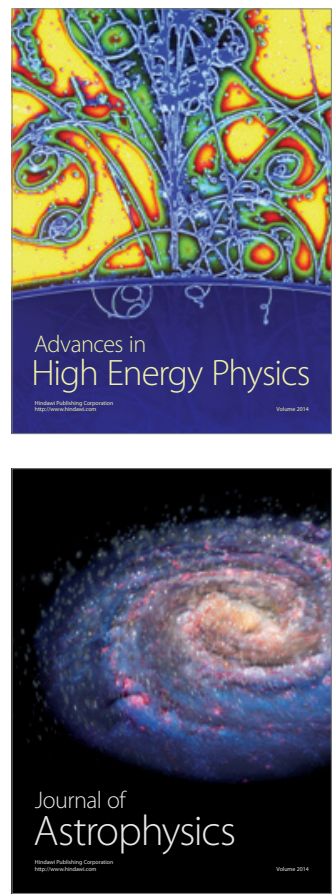
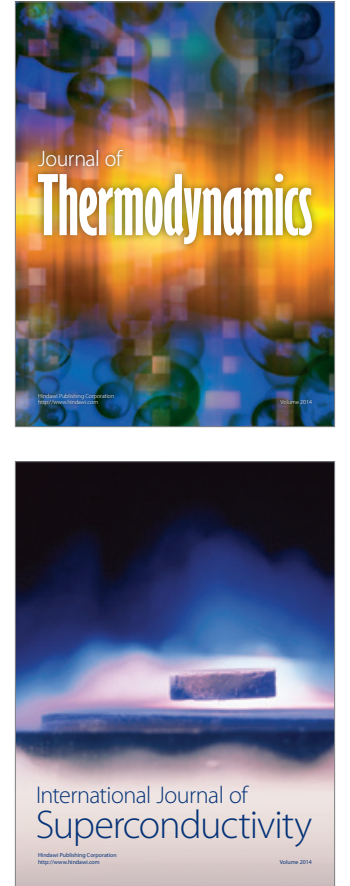
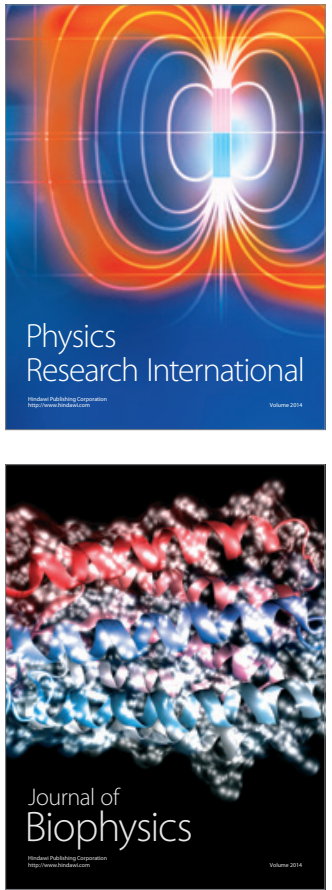
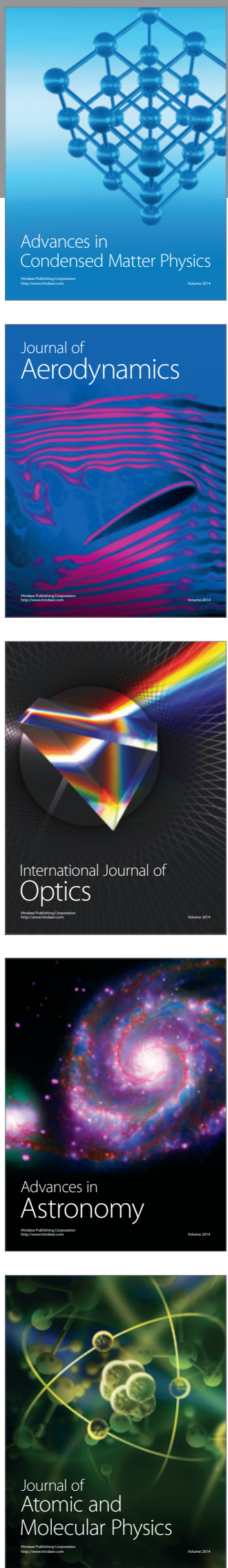